\title{
Encuesta Healthcare Advancement Conference 2016 de Cultura de Seguridad del Paciente en Lima y Callao
}

\section{Healthcare Advancement Conference 2016 survey on Patients Safety Culture in Lima and Callao}

Correspondencia Alejandro Arrieta alejarri@fiu.edu

Recibido: 25/10/2016 Aprobado: 09/11/2016

Citar como: Arrieta-Herrera A, Suárez-Chumacero G, PerezZárate C, Cárdenas-Gamarra $G$, Figueroa-Apéstegui $A$, Cuentas-Jara M, et al. Encuesta Healthcare Advancement Conference 2016 de Cultura de Seguridad del Paciente en Lima y Callao. Acta Med Peru. 2016;33(4):344-5
Alejandro Arrieta-Herrera1', Gabriela Suárez-Chumacero², Cory Perez-Zárate³, Giuliana Cárdenas-Gamarra ${ }^{4}$, Alan Figueroa-Apéstegui ${ }^{5}$, Mariano CuentasJara $^{3}$, Cesar Neves-Catter ${ }^{6}$

1 Departamento de Gestión y Políticas de Salud, Florida International University. Miami, USA.

2 Departamento de Economía, Universidad de Piura. Piura, Perú.

3 Comité de Educación Médica Continúa, Colegio Médico del Perú. Lima, Perú.

4 Comité Nacional de Investigación Científica, Colegio Médico del Perú. Lima, Perú.

5 Asociación Nacional de Médicos Residentes del Perú. Lima, Perú.

6 Programa de Gerencia de Servicios de Salud, Universidad ESAN. Lima, Perú.

\section{Sr. Editor:}

La encuesta Healthcare Advancement Conference (HAC) 2016 representa el primer esfuerzo para medir la cultura de seguridad del paciente en establecimientos de salud públicos y privados de Lima y el Callao. La "cultura de seguridad es el producto de valores, actitudes, percepciones, competencias y patrones de conducta, individuales y grupales, que determinan el compromiso, estilo y capacidad para una gestión segura y saludable de una organización." [i].

La encuesta HAC 2016 consistió en la aplicación de la versión validada en español de la encuesta sobre Cultura de Seguridad del Paciente para hospitales de la Agency for Healthcare Research and Quality (AHRQ), la cual mide la seguridad del paciente de acuerdo a 12 dimensiones, abarcando aspectos de la atención tanto a nivel individual como institucional. La encuesta es de uso extensivo en establecimientos de más de 60 países incluido Estados Unidos ${ }^{[2]}$, España ${ }^{[3]}$, y otros ${ }^{[4]}$.

Entre julio y agosto, más de 1600 profesionales de salud de Lima y el Callao completaron la encuesta HAC 2016 gracias a los esfuerzos de difusión del Colegio Médico del Perú, la Asociación Nacional de Médicos Residentes del Perú, el Colegio de Enfermeros del Perú y la Asociación de Calidad en Salud del Perú. Del total de entrevistados, $50 \%$ eran médicos, $19 \%$ residentes de medicina, $17 \%$ enfermeros y técnicos de enfermería y $14 \%$ eran otros profesionales de salud. Además, según el tipo de institución en la que laboraba el entrevistado, $52 \%$ correspondió a un establecimiento del Ministerio de Salud (MINSA), 25\% a EsSalud, 17\% al sector privado, y $6 \%$ a las Fuerzas Armadas y Policía Nacional.

Los resultados mostrados en la Tabla 1 y disponibles con mayor detalle en http://hac.fiu.edu, muestran que no existe una diferencia estadísticamente significativa entre las dimensiones de seguridad del paciente en establecimientos de EsSalud y MINSA, excepto en el caso de "personal suficiente" " "transferencia de información"; comparado con el sector público, los establecimientos del sector privado recibieron en promedio un mayor porcentaje de respuestas positivas en todas 
Tabla 1. Encuesta HAC 2016. Porcentaje respuestas positivas para cada dimensión de la cultura de seguridad del paciente en Lima y Callao ( $n=1679)$.

\begin{tabular}{|c|c|c|c|c|}
\hline \multirow{2}{*}{ Dimensión } & \multicolumn{4}{|c|}{ Porcentaje de respuesta positiva por institución * } \\
\hline & MINSA & EsSalud & Privado & Total \\
\hline Trabajo en equipo dentro de las unidades & $62 \%$ & $65 \%$ & $76 \% † \ddagger$ & $66 \%$ \\
\hline $\begin{array}{l}\text { Expectativas y acciones del supervisor para promover la } \\
\text { seguridad de los pacientes }\end{array}$ & $47 \%$ & $46 \%$ & $58 \% † \ddagger$ & $49 \%$ \\
\hline Aprendizaje organizativo-Mejorías continuas & $58 \%$ & $58 \%$ & $71 \% \dagger \ddagger$ & $61 \%$ \\
\hline $\begin{array}{l}\text { Apoyo que dan los administradores a la seguridad del } \\
\text { paciente }\end{array}$ & $33 \%$ & $36 \%$ & $63 \% † \ddagger$ & $39 \%$ \\
\hline Comunicación e información sobre errores & $33 \%$ & $34 \%$ & $55 \%$ & $37 \%$ \\
\hline Frecuencia de incidentes reportados & $27 \%$ & $24 \%$ & $47 \% † \ddagger$ & $30 \%$ \\
\hline Percepciones generales de la seguridad del paciente & $39 \%$ & $42 \%$ & $60 \% † \ddagger$ & $44 \%$ \\
\hline Grado al que la comunicación es abierta & $32 \%$ & $34 \%$ & $42 \% \dagger \ddagger$ & $35 \%$ \\
\hline Trabajando en equipo a través de las unidades & $40 \%$ & $39 \%$ & $57 \%+\ddagger$ & $43 \%$ \\
\hline Personal suficiente para manejar la carga de trabajo & $25 \%$ & $27 \% \S$ & $31 \% \ddagger$ & $27 \%$ \\
\hline $\begin{array}{l}\text { Transferencia de información sobre atención al paciente } \\
\text { entre unidades y turnos }\end{array}$ & $36 \%$ & $41 \% \S$ & $55 \% † \ddagger$ & $41 \%$ \\
\hline Respuesta no punitiva a errores & $29 \%$ & $31 \%$ & $33 \% \ddagger$ & $30 \%$ \\
\hline $\begin{array}{l}\text { Grado de seguridad del paciente en la unidad o área de } \\
\text { trabajo (\% Excelente o Muy bueno) }\end{array}$ & $14 \%$ & $13 \%$ & $37 \% \dagger \ddagger$ & $18 \%$ \\
\hline
\end{tabular}

MINSA: Ministerio de Salud. * Respuestas positivas: favorecen la cultura de seguridad del paciente. Los resultados presentan el promedio del porcentaje de personas que tuvieron una respuesta positiva para cada una de las preguntas que componen las dimensiones. Diferencia estadística significativa al $5 \%$ o menos: $†$ hay diferencia entre privado y EsSalud; ¥ hay diferencia entre privado y MINSA; § hay diferencia entre EsSalud y MINSA.

las dimensiones de seguridad del paciente.; el porcentaje de profesionales que calificó el grado de cultura de seguridad del paciente de su unidad de trabajo como excelente o muy bueno fue muy reducido en el caso de establecimientos públicos, muy por debajo de lo obtenido en el sector privado.

La encuesta HAC 2016 revela serias deficiencias en los establecimientos de salud en términos de seguridad del paciente. Este estudio y las siguientes encuestas bianuales HAC pueden proveer la evidencia necesaria para diseñar políticas públicas efectivas orientadas a mejorar la calidad de salud en el país, y pueden servir de punto referencia y comparación a todo establecimiento de salud que implemente la encuesta internamente.

Fuente de financiamiento: Healthcare Management Americas at Florida International University.

Conflicto de intereses: Los autores declaran no tener conflicto de intereses con la publicación de este artículo.

\section{REFERENCIAS BIBLIOGRÁFICAS}

1. RockvilleW, Sorra J, Gray L, Streagle S, FamolaroT, Yount N, et al. AHRQ Hospital Survey on Patient Safety Culture: User's Guide. Rockville, MD: Agency for Healthcare Research and Quality; 2016.

2. Famolaro T, Yount N, Burns W, et al. Hospital Survey on Patient Safety Culture 2016 User Comparative Database Report. (Prepared by Westat, Rockville, MD, under Contract No. HHSA 290201300003C). AHRQ Publication No. 16-0021-EF. Rockville, MD: Agency for Healthcare Research and Quality. 2016.

3. Ministerio de Sanidad y Política Social de España. Análisis de la cultura sobre seguridad del paciente en el ámbito hospitalario del Sistema Nacional de Salud Español. Informes, Estudios e Investigación. Madrid; Ministerio de Sanidad y Política Social; 2009.

4. Wagner C, Smits M, Sorra J, Huang CC. Assessing patient safety culture in hospitals across countries. Int J Qual Health Care. 2013;25(3):213-21. 